\title{
Mistargeting of Peroxisomal EHHADH and Inherited Renal Fanconi's Syndrome
}

Klootwijk, Enriko D ; Reichold, Markus ; Helip-Wooley, Amanda ; Tolaymat, Asad ; Broeker, Carsten ; Robinette, Steven L ; Reinders, Joerg ; Peindl, Dominika ; Renner, Kathrin ; Eberhart, Karin ; Assmann, Nadine ; Oefner, Peter J ; Dettmer, Katja ; Sterner, Christina ; Schroeder, Josef ; Zorger, Niels ; Witzgall, Ralph ; Reinhold, Stephan W ; Stanescu, Horia C ; Bockenhauer, Detlef ; Jaureguiberry, Graciana ; Courtneidge, Holly ; Hall, Andrew M ; Wijeyesekera, Anisha D ; Holmes, Elaine ; Nicholson, Jeremy K ; O’Brien, Kevin ; Bernardini, Isa ; Krasnewich, Donna M ; Arcos-Burgos, Mauricio ; Izumi, Yuichiro ; Nonoguchi, Hiroshi ; Jia, Yuzhi ; Reddy, Janardan K ; Ilyas, Mohammad ; Unwin, Robert J ; Gahl, William A ; Warth, Richard ; Kleta, Robert

\begin{abstract}
Background: In renal Fanconi's syndrome, dysfunction in proximal tubular cells leads to renal losses of water, electrolytes, and low-molecular-weight nutrients. For most types of isolated Fanconi's syndrome, the genetic cause and underlying defect remain unknown. Methods: We clinically and genetically characterized members of a five-generation black family with isolated autosomal dominant Fanconi's syndrome. We performed genomewide linkage analysis, gene sequencing, biochemical and cell-biologic investigations of renal proximal tubular cells, studies in knockout mice, and functional evaluations of mitochondria. Urine was studied with the use of proton nuclear magnetic resonance (1H-NMR) spectroscopy. Results: We linked the phenotype of this family's Fanconi's syndrome to a single locus on chromosome 3q27, where a heterozygous missense mutation in EHHADH segregated with the disease. The p.E3K mutation created a new mitochondrial targeting motif in the N-terminal portion of EHHADH, an enzyme that is involved in peroxisomal oxidation of fatty acids and is expressed in the proximal tubule. Immunocytofluorescence studies showed mistargeting of the mutant EHHADH to mitochondria. Studies of proximal tubular cells revealed impaired mitochondrial oxidative phosphorylation and defects in the transport of fluids and a glucose analogue across the epithelium. 1H-NMR spectroscopy showed elevated levels of mitochondrial metabolites in urine from affected family members. Ehhadh knockout mice showed no abnormalities in renal tubular cells, a finding that indicates a dominant negative nature of the mutation rather than haploinsufficiency. Conclusions: Mistargeting of peroxisomal EHHADH disrupts mitochondrial metabolism and leads to renal Fanconi's syndrome; this indicates a central role of mitochondria in proximal tubular function. The dominant negative effect of the mistargeted protein adds to the spectrum of monogenic mechanisms of Fanconi's syndrome. (Funded by the European Commission Seventh Framework Programme and others.)
\end{abstract}

DOI: https://doi.org/10.1056/NEJMoa1307581

Posted at the Zurich Open Repository and Archive, University of Zurich

ZORA URL: https://doi.org/10.5167/uzh-105862

Journal Article

Published Version 
Originally published at:

Klootwijk, Enriko D; Reichold, Markus; Helip-Wooley, Amanda; Tolaymat, Asad; Broeker, Carsten; Robinette, Steven L; Reinders, Joerg; Peindl, Dominika; Renner, Kathrin; Eberhart, Karin; Assmann, Nadine; Oefner, Peter J; Dettmer, Katja; Sterner, Christina; Schroeder, Josef; Zorger, Niels; Witzgall, Ralph; Reinhold, Stephan W; Stanescu, Horia C; Bockenhauer, Detlef; Jaureguiberry, Graciana; Courtneidge, Holly; Hall, Andrew M; Wijeyesekera, Anisha D; Holmes, Elaine; Nicholson, Jeremy K; O’Brien, Kevin; Bernardini, Isa; Krasnewich, Donna M; Arcos-Burgos, Mauricio; Izumi, Yuichiro; Nonoguchi, Hiroshi; Jia, Yuzhi; Reddy, Janardan K; Ilyas, Mohammad; Unwin, Robert J; Gahl, William A; Warth, Richard; Kleta, Robert (2014). Mistargeting of Peroxisomal EHHADH and Inherited Renal Fanconi's Syndrome. New England Journal of Medicine, 370(2):129-138.

DOI: https://doi.org/10.1056/NEJMoa1307581 


\section{Mistargeting of Peroxisomal EHHADH and Inherited Renal Fanconi's Syndrome}

\author{
Enriko D. Klootwijk, Ph.D., Markus Reichold, Ph.D., Amanda Helip-Wooley, Ph.D., \\ Asad Tolaymat, M.D., Carsten Broeker, M.Sc., Steven L. Robinette, B.Sc., \\ Joerg Reinders, Ph.D., Dominika Peindl, M.D., Kathrin Renner, Ph.D., Karin Eberhart, Ph.D., \\ Nadine Assmann, M.Sc., Peter J. Oefner, M.D., Ph.D., Katja Dettmer, Ph.D., \\ Christina Sterner, M.Sc., Josef Schroeder, Ph.D., Niels Zorger, M.D., Ph.D., \\ Ralph Witzgall, M.D., Ph.D., Stephan W. Reinhold, M.D., Ph.D., \\ Horia C. Stanescu, M.D., Ph.D., Detlef Bockenhauer, M.D., Ph.D., \\ Graciana Jaureguiberry, M.D., Holly Courtneidge, M.Sc., Andrew M. Hall, M.D., Ph.D., \\ Anisha D. Wijeyesekera, Ph.D., Elaine Holmes, Ph.D., Jeremy K. Nicholson, Ph.D., \\ Kevin O'Brien, M.S.N., Isa Bernardini, M.Ed., Donna M. Krasnewich, M.D., Ph.D., \\ Mauricio Arcos-Burgos, M.D., Ph.D., Yuichiro Izumi, M.D., Ph.D., \\ Hiroshi Nonoguchi, M.D., Ph.D., Yuzhi Jia, M.D., Ph.D., Janardan K. Reddy, M.D., \\ Mohammad Ilyas, M.D., Robert J. Unwin, M.D., Ph.D., William A. Gahl, M.D., Ph.D., \\ Richard Warth, M.D., Ph.D., and Robert Kleta, M.D., Ph.D.
}

A BSTRACT

\section{BACKGROUND}

In renal Fanconi's syndrome, dysfunction in proximal tubular cells leads to renal losses of water, electrolytes, and low-molecular-weight nutrients. For most types of isolated Fanconi's syndrome, the genetic cause and underlying defect remain unknown.

\section{METHODS}

We clinically and genetically characterized members of a five-generation black family with isolated autosomal dominant Fanconi's syndrome. We performed genomewide linkage analysis, gene sequencing, biochemical and cell-biologic investigations of renal proximal tubular cells, studies in knockout mice, and functional evaluations of mitochondria. Urine was studied with the use of proton nuclear magnetic resonance ( $\left.{ }^{1} \mathrm{H}-\mathrm{NMR}\right)$ spectroscopy.

RESULTS

We linked the phenotype of this family's Fanconi's syndrome to a single locus on chromosome 3q27, where a heterozygous missense mutation in EHHADH segregated with the disease. The p.E3K mutation created a new mitochondrial targeting motif in the N-terminal portion of EHHADH, an enzyme that is involved in peroxisomal oxidation of fatty acids and is expressed in the proximal tubule. Immunocytofluorescence studies showed mistargeting of the mutant EHHADH to mitochondria. Studies of proximal tubular cells revealed impaired mitochondrial oxidative phosphorylation and defects in the transport of fluids and a glucose analogue across the epithelium. ${ }^{1} \mathrm{H}-\mathrm{NMR}$ spectroscopy showed elevated levels of mitochondrial metabolites in urine from affected family members. Ehhadh knockout mice showed no abnormalities in renal tubular cells, a finding that indicates a dominant negative nature of the mutation rather than haploinsufficiency.

CONCLUSIONS

Mistargeting of peroxisomal EHHADH disrupts mitochondrial metabolism and leads to renal Fanconi's syndrome; this indicates a central role of mitochondria in proximal tubular function. The dominant negative effect of the mistargeted protein adds to the spectrum of monogenic mechanisms of Fanconi's syndrome. (Funded by the European Commission Seventh Framework Programme and others.)
From the Centre for Nephrology (E.D.K., H.C.S., D.B., G.J., H.C., A.M.H., R.J.U., R.K.) and Institute of Child Health (D.B., R.K.), University College London, and Biomolecular Medicine, Imperial College London (S.L.R., A.D.W., E.H., J.K.N.) both in London; the Departments of Medical Cell Biology (M.R., C.B., D.P., C.S., R. Warth), Internal Medicine III (K.R.), Internal Medicine II (S.W.R.), and Molecular and Cellular Anatomy (R. Witzgall) and the Institutes of Functional Genomics (J.R., K.E., N.A., P.J.O., K.D.) and Pathology (J.S.), University of Regensburg, and the Department of Radiology, Barmherzige Brueder Hospital (N.Z.) - all in Regensburg, Germany; the National Human Genome Research Institute (A.H.-W., S.L.R., H.C.S., K.O., I.B., D.M.K., W.A.G., R.K.) and National Heart, Lung, and Blood Institute (Y.I.), National Institutes of Health, Bethesda, MD; the Division of Pediatric Nephrology, University of Florida, Jacksonville (A.T., M.I.); the Genome Biology Department, Australian National University, Canberra, ACT, Australia (M.A.-B.); Kitasato University Medical Center, Saitama, Japan (H.N.); and the Department of Pathology, Northwestern University, Chicago (Y.J., J.K.R.). Address reprint requests to Dr. Kleta at the Centre for Nephrology, University College London, Royal Free Hospital, Rowland Hill St., London NW3 2PF, United Kingdom, or at r.kleta@ucl.ac.uk.

Drs. Klootwijk, Reichold, Helip-Wooley, Gahl, Warth, and Kleta contributed equally to this article.

N Engl J Med 2014;370:129-38. DOI: 10.1056/NEJMoal307581 Copyright @ 2014 Massachusetts Medical Society. 
T HE KIDNEY PLAYS A CRUCIAL ROLE IN maintaining fluid and electrolyte homeostasis. In adults, selective filtration by the renal glomerulus produces more than 150 liters of ultrafiltrate every day, $99 \%$ of which must be reabsorbed by highly specialized segments of the renal tubule. Impaired function of the first postglomerular segment, the proximal tubule, can cause massive and life-threatening losses of fluids, electrolytes, and low-molecular-weight nutrients. Such dysfunction, known as renal Fanconi's syndrome since the early 1900s (for a historical overview, see Kleta and $\mathrm{Gahl}^{1}$ ), most often occurs in children as part of multisystem metabolic diseases such as cystinosis, Dent's disease, the FanconiBickel syndrome, the oculocerebrorenal syndrome (Lowe's syndrome), tyrosinemia type I, Wilson's disease, fructose intolerance, galactosemia, the ARC (arthrogryposis, renal dysfunction, and cholestasis) syndrome, and mitochondrial disorders. In both children and adults, acquired Fanconi's syndrome may result from toxic effects of anti-human immunodeficiency virus drugs, heavy metals, antibiotics, valproic acid, glue sniffing, or exposure to suramin, fumaric acid, or ifosfamide. ${ }^{2,3} \mathrm{Fi}$ nally, there have been rare cases of severe, isolated renal Fanconi's syndrome in which the molecular, biochemical, and cellular defects remain unknown. ${ }^{4}$ Elucidation of these defects might provide a better understanding of the mechanisms of tubular reabsorption and lead to new therapeutic interventions.

One possible cause of renal Fanconi's syndrome involves impaired mitochondrial function, because the high metabolic activity of the proximal tubule makes it particularly susceptible to restrictions in energy output. ${ }^{5}$ Mitochondrial production of ATP, which is essential for generating the energydependent ion gradients that drive renal tubular reabsorption, is impaired in acute kidney injury. ${ }^{6}$ Nevertheless, the mechanism whereby a genetic defect in a mitochondrial protein can damage tubular function has not been delineated. We studied a family with isolated, idiopathic renal Fanconi's syndrome in an effort to identify the molecular, biochemical, and cellular defects that result in impaired renal tubular reabsorption.

\section{METHODS}

\section{PATIENTS}

Seven members of a black family with generalized Fanconi's syndrome were admitted to the
National Institutes of Health Clinical Center and enrolled in a clinical protocol for the diagnosis and treatment of inborn errors of metabolism and other genetic defects. Eleven other family members (two of whom had Fanconi's syndrome) were evaluated locally. All gave written informed consent. The diagnosis of Fanconi's syndrome was established by means of routine laboratory tests of urine and blood samples. All investigations, including genetic studies, were approved by the institutional review board of the National Human Genome Research Institute and were conducted according to the principles of the Declaration of Helsinki. Proton nuclear magnetic resonance ( ${ }^{1} \mathrm{H}$-NMR) spectroscopy ${ }^{7}$ was used to assess urine samples from patients, as compared with samples from unaffected family members (see the Methods section in the Supplementary Appendix, available with the full text of this article at NEJM.org).

\section{GENETIC STUDIES}

DNA was isolated from whole blood with the use of standard procedures and was genotyped with 2000 highly polymorphic sequence-tagged site markers by the company deCODE Genetics. Multipoint parametric linkage analysis was performed with the use of established procedures. ${ }^{8}$ Coding exons and splice sites for all genes within the linked locus were sequenced with the use of traditional Sanger sequencing technology (Transgenomic). Recognized sequence variants were examined for segregation in all nine affected persons and in nine unaffected persons. Sequence variants were tested for their presence or absence in 200 control alleles each from black persons and from white persons (Coriell Institute for Medical Research). Public databases (dbSNP, build 137, and the 1000 Genomes Project, release 12) were also searched. Observed new sequence variants were assessed for uniqueness, conservation in various species, and potential effects on intracellular targeting (see the Methods section in the Supplementary Appendix).

\section{RENAL PROXIMAL TUBULAR CELL LINE}

A permanently transfected, inducible renal proximal tubular cell line derived from LLC-PK1 cells and carrying the identified mutation in EHHADH was created with the use of recombinant technology. ${ }^{9}$ LLC-PK1 cells are an established model and reliably express many properties of the renal proximal tubule. ${ }^{3,10}$ Renal and intracellular localization of EHHADH was studied; EHHADH is 
involved in peroxisomal oxidation of fatty acids and is expressed in the proximal tubule. Fluid transport in the proximal tubule (i.e., formation of fluid-filled domes and transport of specific substrates) was assessed with the use of established techniques. ${ }^{11}$ For electron-microscopic studies and assessment of mitochondrial respiratory function, cells were prepared and investigated with the use of established procedures ${ }^{12,13}$ (see the Methods section in the Supplementary Appendix).

\section{COIMMUNOPRECIPITATION OF EHHADH}

Nonmutant EHHADH and mutant EHHADH were coimmunoprecipitated with HADHA and HADHB (hydroxyacyl-coenzyme A [CoA] dehydrogenase3-ketoacyl-CoA thiolase-enoyl-CoA hydratase), which are parts of the heterodimeric enzyme complex (mitochondrial trifunctional protein), with the use of an anti-HADHB antibody. Immunoblotting was performed with the use of an anti-EHHADH antibody (detailed in the Methods section in the Supplementary Appendix).

\section{Ehhadh KNOCKOUT MICE}

We previously generated Ehhadh knockout mice to study the biochemical function of the peroxisomal protein EHHADH. ${ }^{14}$ The mice were viable and did not have detectable gross phenotypic defects (Fig. S1 in the Supplementary Appendix). Levels of urinary phosphate and urinary metabolites were assessed in knockout and control mice with the use of established analytic procedures. ${ }^{15}$ Bone studies were performed in the mice at 45 and 51 weeks of age. For details of the studies in knockout mice, see the Methods section in the Supplementary Appendix.

\section{RESULTS}

\section{FAMILY STUDIES}

Renal Fanconi's syndrome was inherited in an autosomal dominant fashion in this extended black family (Fig. 1A) and was manifested as rickets (Fig. 1B), impaired growth, glucosuria (Fig. 1C), generalized aminoaciduria, phosphaturia, metabolic acidosis, and low-molecular-weight proteinuria (Table S1 in the Supplementary Appendix). Glomerular function, determined on the basis of creatinine measurements in plasma and 24-hour urine specimens, was normal. ${ }^{16}$ The affected 74-year-old matriarch presented with slightly impaired kidney function (plasma creatinine level, $1.4 \mathrm{mg}$ per deciliter [124 $\mu \mathrm{mol}$ per liter]) but had had normal kidney function at 58 years of age (plasma creatinine level, $1.1 \mathrm{mg}$ per deciliter [ $97 \mu \mathrm{mol}$ per liter]). Urinary protein excretion in affected family members was as high as $1.2 \mathrm{~g}$ per day but was not associated with renal failure. ${ }^{16}$ Affected persons in this family, ranging in age from 1 to 74 years, had uneventful gestational, birth, and infancy histories. The primary clinical manifestation was rickets, due mainly to renal phosphate losses, with bowing of legs from early childhood. All known causes of Fanconi's syndrome had been previously ruled out.

Metabolomic analysis, including univariate significance testing and principal-component analysis, was performed on urine samples from the affected family members and from unaffected family members. Metabolic analysis revealed a unique clustering that differentiated the two groups (Fig. S2 in the Supplementary Appendix). In addition, ${ }^{1} \mathrm{H}$-NMR spectroscopy identified metabolic markers that were indicative of mitochondrial dysfunction (Fig. 1D, and Table S2 in the Supplementary Appendix).

\section{GENETIC ANALYSES}

We performed genomewide linkage analysis and found significant linkage in a single region (LOD score $>3$ ); the disease segregated in an autosomal dominant fashion with a haplotype on chromosome 3q27 (Fig. 2A). That haplotype, shared by all affected family members and not found in any unaffected members, was flanked by markers D3S3583 and D3S2747 (Fig. 2B). By sequencing all known genes (coding and noncoding exons and splice sites) in this linked region, we identified a single heterozygous missense mutation (c.7G $\rightarrow$ A, p.E3K) in EHHADH (Fig. 2C). The mutation segregated completely in all affected persons and was absent from unaffected relatives, black controls, and all available databases. This heterozygous change of a highly conserved glutamate to lysine in the third amino acid position of EHHADH (Fig. 2C and 2D) predicted the de novo creation of an N-terminal mitochondrial targeting motif in this peroxisomal protein (Fig. S3 in the Supplementary Appendix). ${ }^{17}$

\section{CELL STUDies}

We used LLC-PK1 cells that were permanently transfected with normal or mutant EHHADH as a cellular model to study the function and morphology of the proximal tubule. The control (nonmutant) EHHADH localized only to peroxi- 
somes. However, as predicted by our bioinformatic analyses, mutant EHHADH localized to mitochondria as well as to peroxisomes, a finding that was confirmed by colocalization with an established mitochondrial marker. Localization to mitochondria, in addition to peroxi- somes, was also seen in COS-7 and human embryonic kidney 293 (HEK293) cells. Electron microscopy revealed no morphologic abnormalities in the mitochondria of LLC-PK1 cells that were transfected with the mutant EHHADH (Fig. S3 in the Supplementary Appendix).

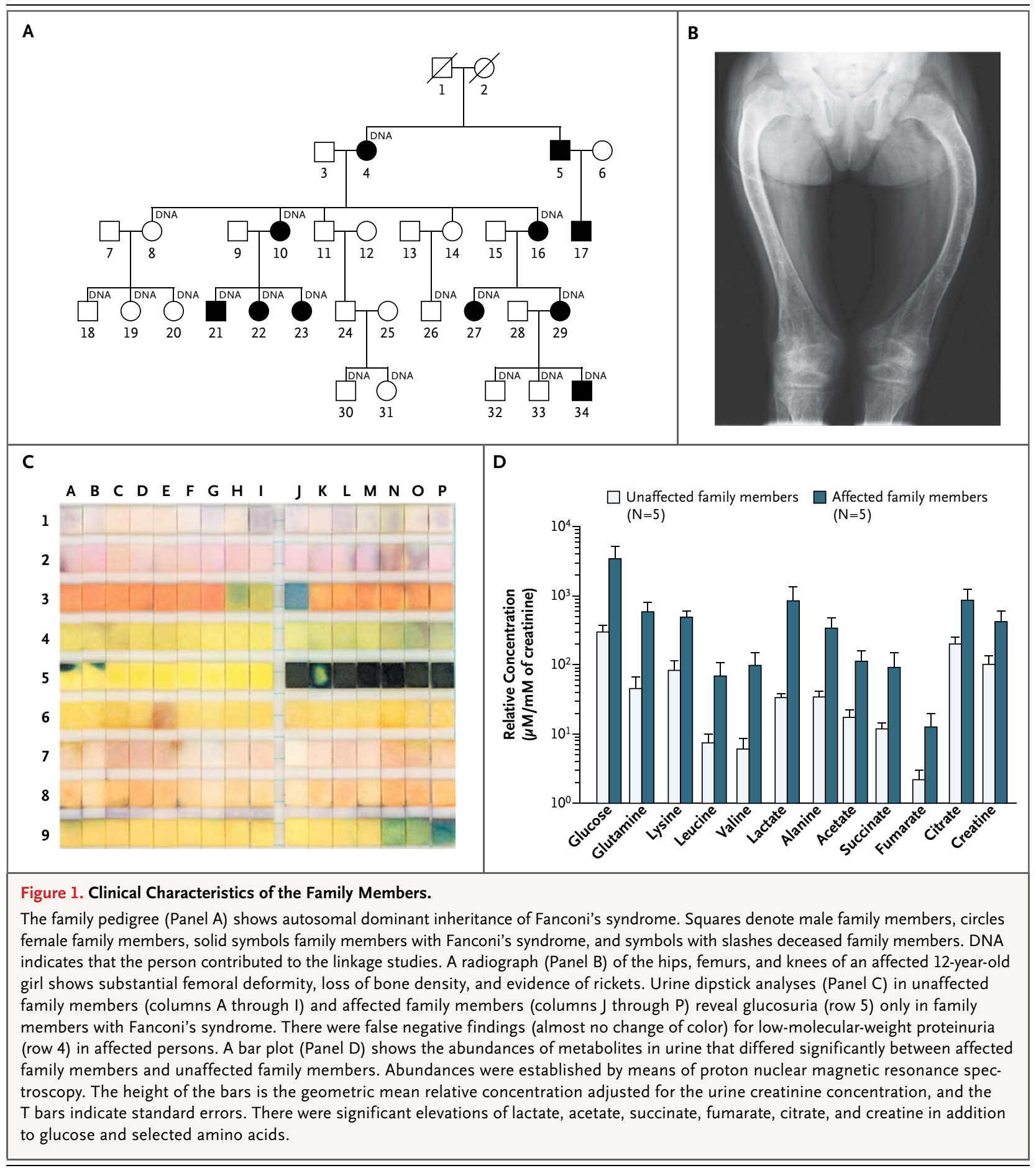


In contrast, permanently transfected renal proximal tubular cells carrying the family's EHHADH mutation exhibited a reproducible and specific defect in the transepithelial transport of fluids, which is a key tubular function. This defect was reflected in the inability to maintain fluid-filled domes in confluent monolayers (Fig. S3 in the Supplementary Appendix); such dome formation is a recognized characteristic of healthy cells. ${ }^{10,18}$ In addition, luminal-to-basolateral transport of methyl $\alpha$-D-glucoside, a surrogate for transepithelial glucose reabsorption, was significantly reduced (Fig. 3A). Furthermore, high-resolution respiratory measurements confirmed an impairment of mitochondrial energy generation. Specifically, the proximal tubular cells that were transfected with the mutant EHHADH, as compared with control EHHADH, showed significantly less capacity for oxidative phosphorylation, a finding that is compatible with an impairment of ATP production (Fig. 3B, and Fig. S4 in the Supplementary Appendix). Mutant EHHADH coimmunoprecipitated with mitochondrial HADHA and HADHB (Fig. 3C), indicating an interaction of the mistargeted EHHADH with mitochondria that is expected to interfere with mitochondrial function.

\section{RENAL IN SITU IMMUNOSTAINING}

In human kidney specimens, strong EHHADH staining was detected in the terminal segments of the proximal tubule, suggesting a role of EHHADH in human kidney physiological processes. In studies of kidney slices obtained from normal mice, immunofluorescence staining showed endogenous EHHADH only in the terminal proximal tubule (Fig. S5 in the Supplementary Appendix).

\section{MOUSE MODELS}

We used Ehhadh-l- mice to determine whether peroxisomal EHHADH haploinsufficiency was likely to have caused Fanconi's syndrome in the family we studied. We did not observe aminoaciduria or glucosuria in these mice (Fig. S6 in the Supplementary Appendix), nor did we observe phosphaturia (phosphate excretion [in micromoles] corrected for urine creatinine concentration [in millimoles], 8.2 \pm 2.7 in five Ehhadh ${ }^{-1-}$ mice and $6.0 \pm 1.2$ in nine control mice; $\mathrm{P}=0.48$ ). We further tested for markers of mitochondrial dysfunction in this knockout model. Mitochondrial dysfunction is often accompanied by elevated excretion of energy inter- mediates, such as lactate and 3-hydroxybutyrate, and impairment of the tricarboxylic acid cycle, with accumulation of succinic acid, fumaric acid, malic acid, and citric acid. In addition, when oxidative phosphorylation is disturbed, cells exhibit increased glycolysis, resulting in elevated levels of glycolytic intermediates (e.g., glyceric acid) as well as an altered lactate:pyruvate ratio. In the Ehhadh knockout mice, however, urinary excretion of these organic compounds was normal (Fig. S6 in the Supplementary Appendix), and the lactate:pyruvate ratio (with both lactate and pyruvate measured in micromoles per millimole of creatinine) was similar to that in control mice (1.33 \pm 0.35 in Ehhadh ${ }^{-1-}$ mice and 1.55 \pm 0.26 in control mice; $\mathrm{P}=0.62$ ). Taken together, these data indicate that a lack of EHHADH had no significant effect on mitochondrial function and suggest that mistargeting of mutant EHHADH to mitochondria, in a dominant negative fashion, is the disease-causing mechanism.

\section{DISCUSSION}

Renal Fanconi's syndrome comprises a heterogeneous group of disorders characterized by proximal tubular dysfunction leading to generalized aminoaciduria, glucosuria, phosphaturia, lowmolecular-weight proteinuria, and metabolic acidosis due to renal bicarbonate losses..$^{19}$ When renal Fanconi's syndrome occurs as a result of multisystem disease, early diagnosis may facilitate therapy directed at the basic defect. ${ }^{20}$ When Fanconi's syndrome is isolated, however, treatment is largely symptomatic because the basic defect is generally unknown. Exceptions include genetic disorders causing a partial Fanconi's syndrome ${ }^{4,21}$ (e.g., renal glucosuria due to biallelic SGLT2 mutations $^{22}$ and phosphaturia associated with autosomal recessive deficiency of phosphate transport ${ }^{23}$ ). Our study elucidates the molecular basis of renal Fanconi's syndrome in a family.

Over time, several hypotheses concerning the defect in proximal tubular function in Fanconi's syndrome have been proposed, including changes in membrane fluidity, increased paracellular permeability with increased back flux, decreased luminal uptake, and decreased basolateral transport from within the cell to the blood or increased back flux from the blood toward the cells. ${ }^{24}$ In Fanconi's syndrome that is due to systemic disease, renal tubular cells may be de- 


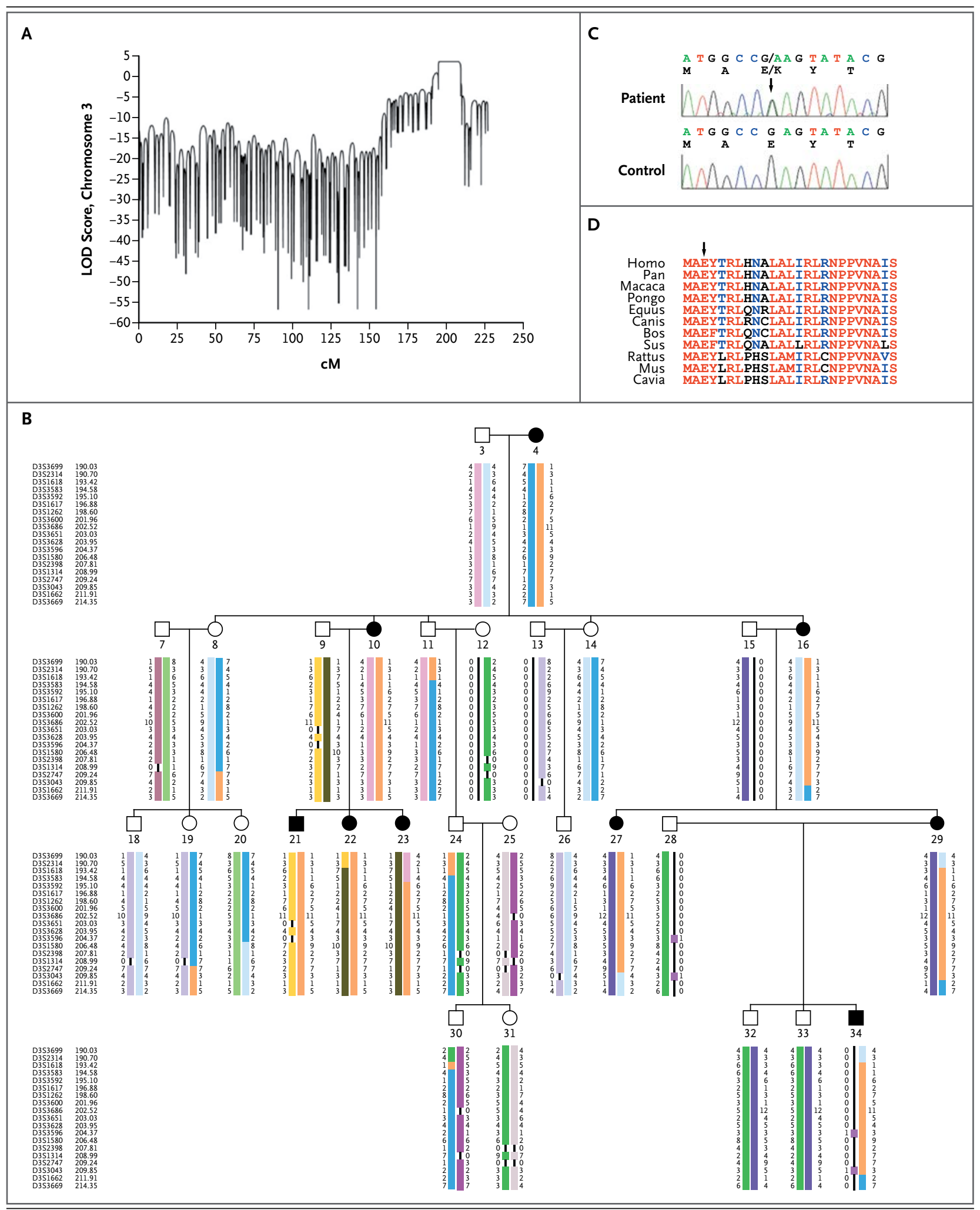


Figure 2 (facing page). Results of Linkage Studies and Sequence Analysis.

Panel A shows the results of multipoint parametric linkage analysis for chromosome 3 . There was distinct and significant linkage (LOD score, 3.61 ) in the region of $200 \mathrm{cM}$. Panel B shows a haplotype reconstruction for the family at this locus, with segregation of the disease allele (shown in orange; D3S3583-D3S2747) in all affected family members. Other colors represent other haplotypes. Panel $\mathrm{C}$ shows original sequence chromatograms for a portion of exon 1 of EHHADH for an affected family member (patient) and her unaffected offspring (control). There is a heterozygous missense mutation, c.7G $\rightarrow$ A (GAG $\rightarrow$ AAG; p.E3K), in the patient's chromatogram (arrow). Panel D shows a protein-alignment (homology) plot of the first 25 amino acids of EHHADH in 11 vertebrate genera, with complete conservation of the E3 locus (arrow).

stroyed directly. In isolated Fanconi's syndrome resulting from a genetic defect, energy production in proximal tubular cells may be impaired, impeding the capacity for reabsorption of lowmolecular-weight proteins.

Remarkably, proximal tubular cells do not use glucose for their energy production ${ }^{25}$; fatty acid oxidation is the predominant energy source. Moreover, mitochondria rather than peroxisomes perform most of this function in proximal tubular cells. Indeed, patients with typical mitochondrial disorders often have Fanconi's syndrome as part of their disease. ${ }^{26}$ Despite widespread acceptance of the requirement of mitochondrial function for renal tubular reabsorption, however, isolated cases of Fanconi's syndrome resulting from mitochondrial dysfunction have not been noted.

Our studies involving a family with renal Fanconi's syndrome and severe rickets provided an opportunity to examine this functional relationship. Affected family members had generalized renal Fanconi's syndrome involving defective reabsorption of phosphate, glucose, bicarbonate, amino acids, water, and low-molecular-weight proteins. They also had a pathogenic mutation (p.E3K) in EHHADH.

This gene encodes the bifunctional peroxisomal enzyme enoyl-CoAhydratase-L-3-hydroxyacyl-CoA dehydrogenase, also referred to as L-bifunctional enzyme or L-PBE, which functions primarily in peroxisomal beta-oxidation of straight-chain saturated acyl-CoA fatty acids ${ }^{27,28}$ and can be induced by ligands of the nuclear receptor peroxi- some proliferator-activated receptor $\alpha$ (PPAR- $\alpha$ ). The first and rate-limiting step of the beta-oxidation pathway is catalyzed by the fatty acid acyl-CoA oxidase, and the second and third reactions are carried out by EHHADH. The fourth and final step is catalyzed by 3-ketoacyl-CoA thiolase. A bifunctional enzyme called D-bifunctional enzyme, which is functionally homologous to EHHADH, also provides for the second and the third steps of the beta-oxidation pathway as a noninducible system acting on branched-chain acyl-CoA fatty acids.

Despite these known roles of L-PBE in peroxisomal oxidation pathways, it plays a minor role in overall fatty acid oxidation and energy production, as indicated by the fact that Ehhadh knockout mice are viable and ostensibly unaffected. ${ }^{29}$ In the Ehhadh ${ }^{-1-}$ mice that we studied, classic peroxisomal beta-oxidation was disrupted owing to removal of exon 4 in the L-PBE gene. ${ }^{14}$ Nevertheless, these mice were fertile and had no gross phenotypic defects, suggesting that their mitochondria provided sufficient energy for growth and development. Specifically, proximal tubular function was not impaired in the Ehhadh knockout mice, as shown by the absence of glucosuria, phosphaturia, and aminoaciduria. Moreover, mitochondrial function appeared to be preserved, with normal urinary excretion of organic acids and normal levels of glycolytic intermediates.

These findings argue against a haploinsufficiency model of disease in our patients and argue for a dominant negative effect of the mutant allele on proximal tubular reabsorption. In particular, the p.E3K mutation in EHHADH targeted the mutant protein mainly to mitochondria instead of peroxisomes (Fig. S3 in the Supplementary Appendix), as a result of the creation of a new targeting signal in the N-terminal of the protein. This finding is consistent with the general consensus that targeting motifs for mitochondrial proteins often occur at the $\mathrm{N}$-terminal of the protein and that targeting motifs for peroxisomal proteins occur at the C-terminal of the protein. Mistargeting of a peroxisomal protein to mitochondria occurs in another metabolic disease, autosomal recessive primary hyperoxaluria. ${ }^{30}$ In that case, however, the cause of the disease is an absence of peroxisomal enzymatic activity.

Because mitochondria are critical for energy production in renal proximal tubules, we investi- 


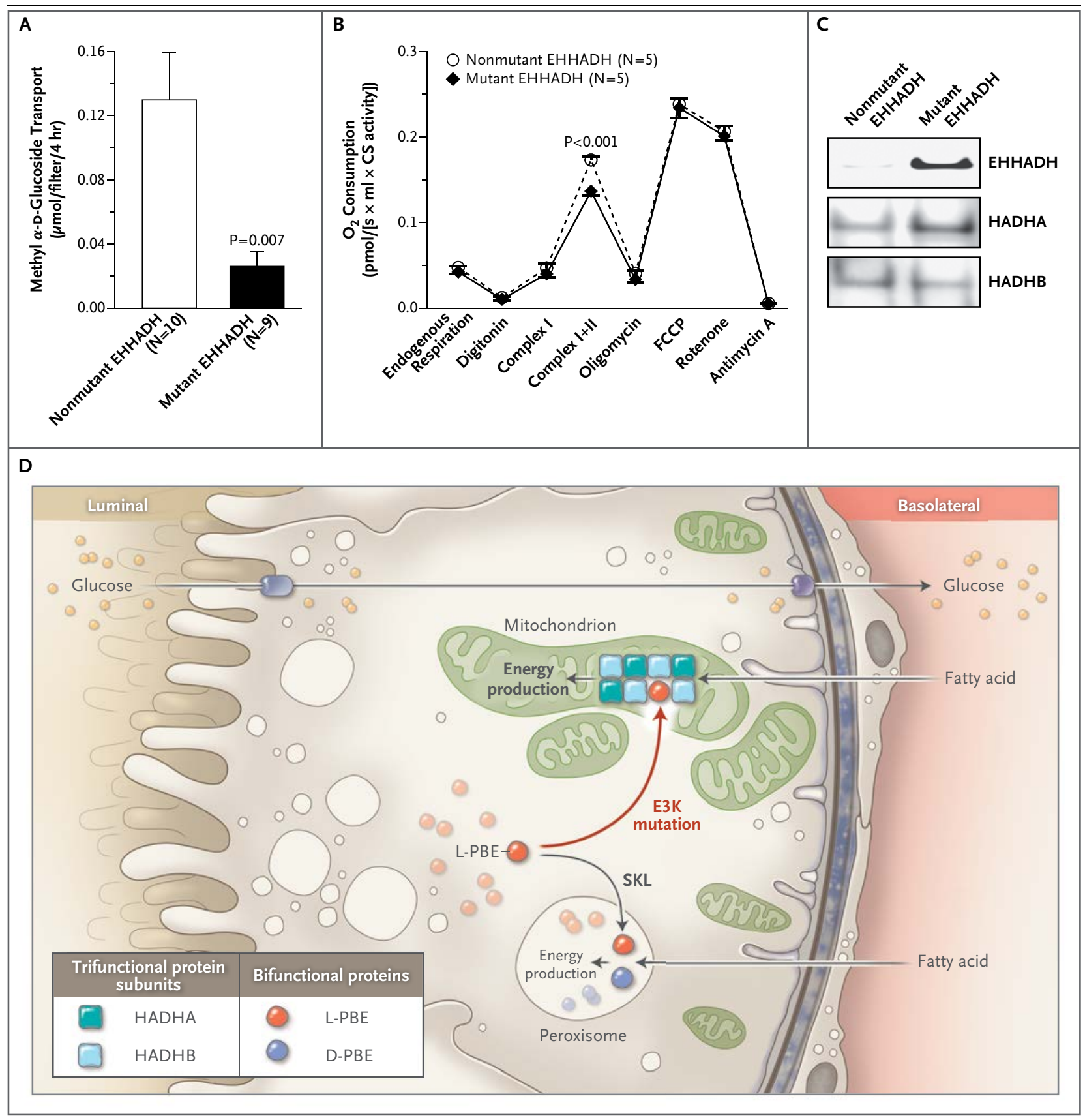

gated the effect of misdirected mutant EHHADH on mitochondrial function. Respirometric measurements of cells expressing mutant EHHADH showed a reduced capacity of the oxidative phosphorylation pathway, and ${ }^{1} \mathrm{H}-\mathrm{NMR}$ spectroscopy of urine specimens from affected family members showed specific elevations of mitochondrial metabolites.

Our data suggest that the isolated complexes of the respiratory chain are working normally but that the activity of the phosphorylation system (e.g., function of ATP synthase) becomes rate-limiting. One possible mechanism for the dominant negative effect of a mutant EHHADH protein on mitochondrial function involves impaired beta-oxidation of fatty acids. There is a high degree of homology between the peroxisomal bifunctional protein (EHHADH) and the 
Figure 3 (facing page). Results of Functional Studies in Cell Culture.

Panel A shows the results of transport studies in inducible, permanently transfected LLC-PKI cells expressing nonmutant or mutant EHHADH. Cells were grown on permeable supports, and the nonmetabolizable glucose surrogate methyl $\alpha$-D-glucoside was used as substrate for $\mathrm{Na}^{+}$-coupled glucose transport. Cells overexpressing the mutant EHHADH exhibited a significantly reduced transport capacity as compared with cells overexpressing the nonmutant protein. I bars indicate standard errors (SE). Panel B shows the results of a high-resolution respirometry experiment in inducible, permanently transfected LLC-PKI cells expressing nonmutant or mutant EHHADH. Oxygen consumption of mitochondria was determined as an indirect measure of oxidative phosphorylation. No significant differences in endogenous respiration were observed in intact cells. After permeabilization of the cell membrane with digitonin and the addition of saturating substrates for complex I, complex II, and adenosine diphosphate, cells expressing mutant EHHADH had a significantly reduced capacity for oxidative phosphorylation $(P<0.001)$. After inhibition of the ATP synthase (with oligomycin) and uncoupling (with p-trifluoromethoxy carbonyl cyanide phenyl hydrazone [FCCP]), the difference in oxygen consumption was abolished, pointing toward a dysfunction in ATP production. For details, see the Methods section and Figure S4 in the Supplementary Appendix. Values are means $\pm S E$, normalized to citrate synthase (CS) activity. Panel $C$ shows the results of coimmunoprecipitation of EHHADH with HADHA and HADHB (hydroxyacyl-coenzyme A [CoA] dehydrogenase-3-ketoacyl-CoA thiolase-enoyl-CoA hydratase) with the use of an anti-HADHB antibody. Immunoblotting against EHHADH showed a distinct band for the mutant EHHADH, indicating that the mutant protein is incorporated into the mitochondrial trifunctional enzyme, whereas the absence of a band for the nonmutant protein indicates negligible incorporation. Immunoblotting against the "bait" protein HADHB and its known interactor, HADHA, showed similar intensities in LLC-PKl cells expressing nonmutant EHHADH and those expressing mutant EHHADH. Panel D shows a proposed model of a dominant negative effect of mutant peroxisomal L-bifunctional enzyme (L-PBE) (EHHADH) disrupting the mitochondrial trifunctional protein hetero-octamer (composed of HADHA and HADHB) in the renal proximal tubule. Glucose is reabsorbed from the luminal (urine-side) membranes to the basolateral (blood-side) membranes and is not used for energy production. Most of the basolateral fatty acid uptake is used for mitochondrial energy production by means of trifunctional protein (HADHA and HADHB). A small fraction of the fatty acid uptake is used for peroxisomal energy production by means of bifunctional protein (D-bifunctional enzyme [D-PBE]). The physiological targeting motif, SKL, normally directs L-PBE to the peroxisome. The E3K mutation instead creates a targeting motif that directs L-PBE to the mitochondrion, where its homology causes it to interfere with the assembly and function of trifunctional protein.

mitochondrial trifunctional protein, which plays the dominant role in overall energy production. Because the mitochondrial trifunctional protein is a heterodimeric octamer, the mutant EHHADH (which, after import and loss of its leader sequence, shows high homology to the mitochondrial trifunctional protein) appears to interact with normal mitochondrial subunits of the mitochondrial trifunctional protein and to functionally disrupt some of these multimers (Fig. 3C and 3D). The extent of dysfunction is dependent on the ratio of abnormal to normal subunits and the affinity of the mutant EHHADH protein for octameric partners. In any event, there appears to be sufficient disruption of mitochondrial energy metabolism to impair reabsorption in the renal proximal tubules, as indicated by our clinical findings and our in vivo and in vitro studies. However, the possibility of an additional dominant negative effect of the mutant EHHADH on peroxisomal function cannot be excluded.

The findings in this family indicate that a monogenic defect leading to intracellular mistargeting of a mutant protein can result in mito- chondrial damage and isolated organ disease - in this case, renal Fanconi's syndrome. ${ }^{31}$ The explanation lies in the unique and particular energy requirements of proximal tubular cells for fatty acid oxidation, combined with the singular disruption of mitochondrial beta-oxidation by the mistargeting of a mutated peroxisomal protein. The elucidation of this defect allows for molecular screening of other family members and the possibility of early intervention to prevent rickets.

\footnotetext{
Supported by grants from the European Commission Seventh Framework Programme (2012-305608 [European Consortium for High-Throughput Research in Rare Kidney Diseases], to Drs. Bockenhauer, Unwin, and Kleta), the David and Elaine Potter Foundation (to Dr. Kleta), St. Peter's Trust for Kidney, Bladder, and Prostate Research (to Drs. Bockenhauer and Kleta), Kids Kidney Research (to Drs. Bockenhauer and Kleta), the Lowe Syndrome Trust (to Drs. Bockenhauer, Unwin, and Kleta), BayGene (to Dr. Oefner), and Deutsche Forschungsgemeinschaft (SFB699, to Drs. Reichold, Reinders, and Warth). Also supported in part by the Intramural Research Program of the National Human Genome Research Institute.

Disclosure forms provided by the authors are available with the full text of this article at NEJM.org.

We thank Drs. Anselm Zdebik and Naomi Issler, both at University College London, for their expert discussions and advice, and Dr. Michael Wiesener, University of Erlangen-Nuremberg, for providing normal human kidney-biopsy specimens.
} 
1. Kleta R, Gahl WA. Cystinosis: antibodies and healthy bodies. J Am Soc Nephrol 2002;13:2189-91.

2. Hall AM, Hendry BM, Nitsch D, Connolly JO. Tenofovir-associated kidney toxicity in HIV-infected patients: a review of the evidence. Am J Kidney Dis 2011;57: 773-80.

3. Ciarimboli G, Holle SK, Vollenbröcker $B$, et al. New clues for nephrotoxicity induced by ifosfamide: preferential renal uptake via the human organic cation transporter 2. Mol Pharm 2011;8:270-9.

4. Kleta R. Fanconi or not Fanconi? Lowe syndrome revisited. Clin J Am Soc Nephrol 2008;3:1244-5.

5. Szeto HH, Liu S, Soong Y, et al. Mitochondria-targeted peptide accelerates ATP recovery and reduces ischemic kidney injury. J Am Soc Nephrol 2011;22:1041-52. 6. Hall AM. Pores for thought: new strategies to re-energize stressed mitochondria in acute kidney injury. J Am Soc Nephrol 2011;22:986-9.

7. Vilasi A, Cutillas PR, Maher AD, et al. Combined proteomic and metabonomic studies in three genetic forms of the renal Fanconi syndrome. Am J Physiol Renal Physiol 2007;293:F456-F467.

8. Landouré G, Zdebik AA, Martinez TL, et al. Mutations in TRPV4 cause CharcotMarie-Tooth disease type 2C. Nat Genet 2010;42:170-4.

9. Izumi $Y$, Nakayama $Y$, Mori $T$, et al. Downregulation of vasopressin V2 receptor promoter activity via V1a receptor pathway. Am J Physiol Renal Physiol 2007;292:F1418F1426.

10. Kleta R, Mohrmann M, Schlatter E. Effects of cell differentiation on ion conductances and membrane voltage in LLC-PK1 cells. Pflugers Arch 1995;429:370-7.

11. Dettmer K, Nürnberger N, Kaspar H, Gruber MA, Almstetter MF, Oefner PJ. Metabolite extraction from adherently growing mammalian cells for metabolomics studies: optimization of harvesting and extraction protocols. Anal Bioanal Chem 2011;399:1127-39.

12. Stadlmann S, Renner K, Pollheimer J, et al. Preserved coupling of oxidative phosphorylation but decreased mitochondrial respiratory capacity in IL-1beta-treated human peritoneal mesothelial cells. Cell Biochem Biophys 2006;44:179-86.

13. Pesta D, Gnaiger E. High-resolution respirometry: OXPHOS protocols for human cell cultures and permeabilized fibers from small biopsies of human muscle. Methods Mol Biol 2012;810:25-58.

14. Qi C, Zhu Y, Pan J, et al. Absence of spontaneous peroxisome proliferation in enoyl-CoA hydratase/L-3-hydroxyacyl-CoA dehydrogenase-deficient mouse liver: further support for the role of fatty acyl CoA oxidase in PPARalpha ligand metabolism. J Biol Chem 1999;274:15775-80.

15. Kaspar H, Dettmer K, Gronwald W, Oefner PJ. Automated GC-MS analysis of free amino acids in biological fluids. J Chromatogr B Analyt Technol Biomed Life Sci 2008;870:222-32.

16. Tolaymat A, Sakarcan A, Neiberger R. Idiopathic Fanconi syndrome in a family. Part I: clinical aspects. J Am Soc Nephrol 1992;2:1310-7.

17. Claros MG, Vincens P. Computational method to predict mitochondrially imported proteins and their targeting sequences. Eur J Biochem 1996;241:779-86.

18. Hull RN, Cherry WR, Weaver GW. The origin and characteristics of a pig kidney cell strain, LLC-PK. In Vitro 1976;12:6707.

19. Kleta R, Bockenhauer D. Bartter syndromes and other salt-losing tubulopathies. Nephron Physiol 2006;104:p73-p80. 20. Kleta R, Bernardini I, Ueda $M$, et al. Long-term follow-up of well-treated nephropathic cystinosis patients. J Pediatr 2004, 145:555-60.

21. Bockenhauer D, Bokenkamp A, van't Hoff W, et al. Renal phenotype in Lowe syndrome: a selective proximal tubular dysfunction. Clin J Am Soc Nephrol 2008; 3:1430-6.

22. Magen D, Sprecher E, Zelikovic I, Skorecki K. A novel missense mutation in SLC5A2 encoding SGLT2 underlies autosomal-recessive renal glucosuria and aminoaciduria. Kidney Int 2005;67:34-41. 23. Magen D, Berger L, Coady MJ, et al. A loss-of-function mutation in NaPi-IIa and renal Fanconi's syndrome. N Engl J Med 2010;362:1102-9.

24. Foreman JW, Roth KS. Human renal Fanconi syndrome - then and now. Nephron 1989;51:301-6.

25. Balaban RS, Mandel LJ. Metabolic substrate utilization by rabbit proximal tubule: an NADH fluorescence study. Am J Physiol 1988;254:F407-F416.

26. Niaudet P, Rötig A. Renal involvement in mitochondrial cytopathies. Pediatr Nephrol 1996;10:368-73.

27. Reddy JK, Hashimoto T. Peroxisomal beta-oxidation and peroxisome proliferatoractivated receptor alpha: an adaptive metabolic system. Annu Rev Nutr 2001;21:193230.

28. Wanders RJ, Vreken P, Ferdinandusse S, et al. Peroxisomal fatty acid alpha- and beta-oxidation in humans: enzymology, peroxisomal metabolite transporters and peroxisomal diseases. Biochem Soc Trans 2001;29:250-67.

29. Houten SM, Denis S, Argmann CA, et al. Peroxisomal L-bifunctional enzyme (Ehhadh) is essential for the production of medium-chain dicarboxylic acids. J Lipid Res 2012;53:1296-303.

30. Danpure CJ. Primary hyperoxaluria type 1: AGT mistargeting highlights the fundamental differences between the peroxisomal and mitochondrial protein import pathways. Biochim Biophys Acta 2006; 1763:1776-84.

31. Koopman WJ, Willems PH, Smeitink JA. Monogenic mitochondrial disorders. N Engl J Med 2012;366:1132-41.

Copyright (c) 2014 Massachusetts Medical Society.

\section{AN NEJM APP FOR IPHONE}

The NEJM Image Challenge app brings a popular online feature to the smartphone. Optimized for viewing on the iPhone and iPod Touch, the Image Challenge app lets you test your diagnostic skills anytime, anywhere. The Image Challenge app randomly selects from 300 challenging clinical photos published in NEJM, with a new image added each week. View an image, choose your answer, get immediate feedback, and see how others answered.

The Image Challenge app is available at the iTunes App Store. 